\title{
Hormones in infant rhesus monkeys' (Macaca mulatta) hair at birth provide a window into the fetal environment
}

\author{
Amita Kapoor ${ }^{1}$, Gabriele Lubach ${ }^{2,3}$, Curtis Hedman ${ }^{1,4}$, Toni E. Ziegler ${ }^{1}$ and Christopher L. Coe ${ }^{2,3}$
}

BACKGROUND: It is established that maternal parity can affect infant growth and risk for several disorders, but the prenatal endocrine milieu that contributes to these outcomes is still largely unknown. Recently, it has been shown that hormones deposited in hair can provide a retrospective reflection of hormone levels while the hair was growing. Taking advantage of this finding, our study utilized hair at birth to investigate if maternal parity affected fetal hormone exposure during late gestation.

METHODS: Hair was collected from primiparous and multiparous mother and infant monkeys at birth and used to determine steroid hormones embedded in hair while the infant was in utero. A high-pressure liquid chromatography-triple quadrupole mass spectrometry technique was refined, which enabled the simultaneous measurement of eight hormones.

RESULTS: Hormone concentrations were dramatically higher in neonatal compared to maternal hair, reflecting extended fetal exposure as the first hair was growing. Further, hair cortisone was higher in primiparous mothers and infants when compared to the multiparous dyads.

CONCLUSION: This research demonstrates that infant hair can be used to track fetal hormone exposure and a panel of steroid hormones can be quantified from hair specimens. Given the utility in nonhuman primates, this approach can be translated to a clinical setting with human infants.

$\mathbf{T}$ he important influence of maternal age and parity on pregnancy and delivery outcomes is well known (1), but the degree to which a young primiparous female provides a different physiological milieu for the developing fetus is not as appreciated. For example, adolescent females may be more challenged by the energetic demands of pregnancy if they are still growing. The potential conflict between maternal and fetal needs becomes manifest in the gestational anemia that is more common among young mothers, which also increases the likelihood of a reduced placental transfer of iron during the third trimester (2). Several placental hormones, including chorionic gonadotrophin, estrogen, and progesterone have also been reported to be higher during early pregnancy in primigravidae (3), and higher levels of prolactin are required during early lactation to induce milk production in first-time mothers (4). To further investigate how parity affects the prenatal endocrine milieu, a novel approach was employed in the following study to examine hormone levels present in infant and maternal hair at birth. Several papers had recently demonstrated that hair hormone levels provide a reliable record of endogenous endocrine activity over the prior 1-3 mo, including in pregnant women $(5,6)$. However, our research is the first to utilize this strategy to retrospectively evaluate fetal hormone exposure. We also broadened the analysis from cortisol to include other steroidal hormones that become incorporated in hair.

The possibility that gravidity engenders different in utero hormone conditions for the fetus has significant implications for postnatal development given the pervasive programming of many physiological regulatory systems that occurs during the prenatal period. In addition to the effects of fetal growth on later health documented by Barker and colleagues (7), many studies have shown that exposure to high levels of glucocorticoids can have a lasting impact on important brain areas, including on the hippocampus. In addition, elevated maternal cortisol during pregnancy has been associated with impairments in neuromotor reflexes and increased emotional reactivity in childhood (8). The levels of glucocorticoid hormone present in maternal and neonatal hair of rhesus monkeys were assessed during the first days after delivery, with the primary aim of comparing infants of primiparous and multiparous females. Because it is known that a significant portion of cortisol is enzymatically converted to cortisone by placental $11-\beta$ hydroxysteroid dehydrogenase (11 $\beta$-HSD), mass spectrometry techniques were refined to quantify both hormones, with the a priori hypothesis that cortisone levels would be higher in infant hair (9). In addition, it was known from prior research on human hair that the follicle also synthesizes 11ß-HSD, which results in the substantial conversion and integration of cortisone into the hair shaft (10).

The novel mass spectrometry method we developed also permitted the simultaneous determination of six other steroid hormones of adrenal, gonadal, and placental origin. It has been well known for over $50 \mathrm{y}$ that prenatal exposure to androgens and estrogens is essential for normal sexual differentiation of

'Wisconsin National Primate Research Center, University of Wisconsin-Madison, Madison, Wisconsin; ${ }^{2}$ Department of Psychology, University of Wisconsin-Madison, Madison, Wisconsin; ${ }^{3}$ Harlow Center for Biological Psychology, University of Wisconsin-Madison, Madison, Wisconsin; ${ }^{4}$ Wisconsin State Laboratory of Hygiene, Madison, Wisconsin. Correspondence: Amita Kapoor (akapoor@wisc.edu) 
the fetus and the emergence of gender differences in appearance and behavior after birth (11). Thus, by assessing the hair of neonates, it may be possible to ascertain more specifically if the prenatal hormone exposure of male and female fetuses is differentially affected by the parity of their mothers. Although it has not been systematically examined in mammalian species, there is considerable precedent in reptiles and birds for a differential secretion of sex-specific hormones based on both the order in which the eggs are laid and ambient environmental conditions $(12,13)$.

The hair of monkeys at birth offers a unique, summative picture of hormone exposure during the final months of gestation because it first begins to grow $\sim 2$ mo before term (14). Previous research on the hair of older monkeys demonstrated that individual differences in hair cortisol levels are relatively stable and further that hair collected after a sustained period of stress reflects the increased secretion of adrenal hormones (6). In addition, hair is a commonly used matrix in forensic analyses, routinely employed for detecting anabolic steroid use by athletes, and used to screen for drugs of abuse and environmental xenobiotics (15-18). Analysis of neonatal hair can reveal exposure to environmental toxicants (19) and a history of maternal cocaine use during pregnancy (20). Another virtue of hair specimens is that the collection is noninvasive and hair can be stored at room temperature for extended periods of time without deterioration of the integrated hormone (21).

Our hair analyte study investigated whether neonatal hair provides a retrospective window on in utero hormone exposure. We quantified both the typical steroid hormones predominant in adult circulation and secondary estrogens, androgens, and corticosteroids, in anticipation from human and guinea pig hair studies that the latter hormones would be present at higher concentrations (9). The major finding is that hair collected from the newborn infant can be utilized to assess in utero hormone exposure. Using our multianalyte approach, a single hair sample provided information on eight steroid hormones. Further, cortisone levels were significantly higher in the hair of both primiparous mothers and their infants than in identically collected hair from multiparous dams and offspring. In keeping with this strong effect of parity, male and female offspring gestated by primiparous dams also had higher levels of estrone and testosterone embedded in their hair, which has important implications for the gender differentiation of their physiology and later behavior.

\section{RESULTS}

Parity Affected Hair Hormone Levels in Both Mother and Infant Monkeys

All infants were born after normal term pregnancies and unassisted vaginal deliveries. The mean gestation length was typical for the species at $169.5 \pm 2.6 \mathrm{~d}$ and was not significantly affected by parity. Infants from primiparous mothers tended to be born at a slightly higher birth weight compared to multiparous infants (primiparous: $515.8 \pm 68.8$ vs. multiparous: $475.6 \pm 52.0 \mathrm{~g} ; P=0.08$ ), but birth weight was not associated with any hair hormone concentrations. To minimize the disturbance of collection procedures on the parturient dam, hair was collected primarily between 2 and $4 \mathrm{~d}$ after delivery (mean $3.29 \mathrm{~d}$ after birth).

Primiparous mothers had significantly higher cortisone levels in their hair than found in multiparous dams (Figure 1a; $P<0.01)$ resulting in a lower hair cortisol:cortisone ratio (Figure 1c; $P<0.05$ ).Maternal hair cortisol levels were not affected by parity (Figure $\mathbf{1 b}$ ).

The hair of infants born to primiparous mothers also had higher cortisone levels (Figure 2a; $P<0.01$ ), and the infants' hair cortisone levels were significantly correlated with their mothers' cortisone levels $(r=0.43 ; P<0.05)$. There was no effect of maternal parity on the infant hair cortisol levels and the hair cortisol/cortisone ratio was similar to the mothers, with the primiparous infants exhibiting a lower ratio $(P<0.01)$. Cortisol values in infant and maternal hair were not correlated $(r=0.14$; NS). A similar effect of maternal parity was evident on the infants' hair estrogens and testosterone concentrations. Neonatal hair from primigravidae had higher estrone compared to the infants of multiparous mothers (Figure $2 \mathrm{~b} ; \mathrm{P}<$ 0.01 ). Both male and female infants from primiparous mothers also had higher levels of testosterone in their hair at birth than did infants from multiparous pregnancies (Figure 2c; $P<0.05$ ).

\section{Successful Implementation of the Hair Hormone Strategy}

The mass spectrometry analytic methods enabled us to detect eight different steroid hormones, permitting the simultaneous assay of glucocorticoids, estrogens, androgens, and
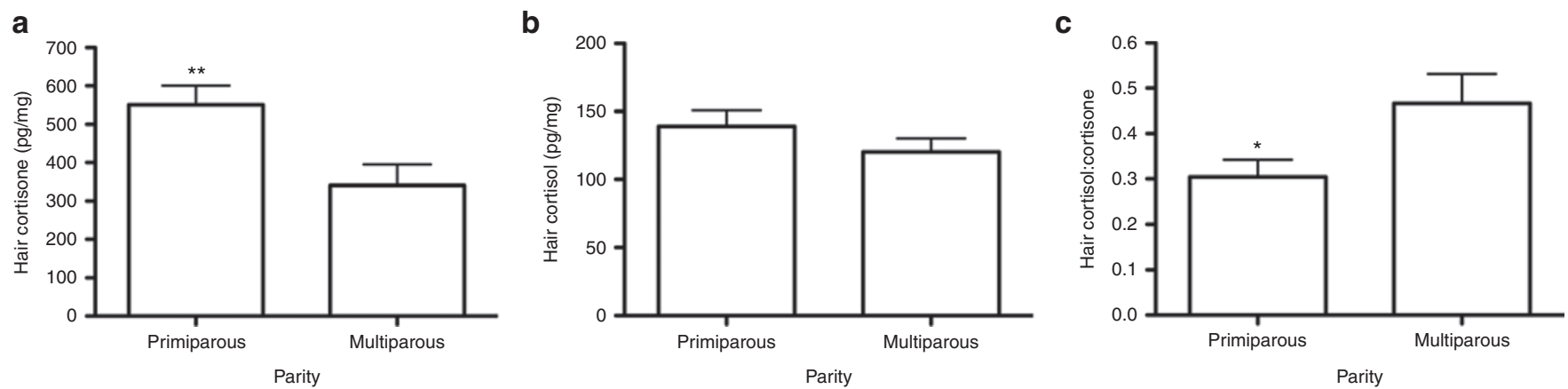

Figure 1. Hair hormone levels in rhesus monkey mothers. Hair cortisone (mean $\pm \mathrm{SEM}$ ) (a), cortisol (b), and (c) cortisol:cortisone ratio from primiparous $(n=19)$ and multiparous $(n=16)$ postpartum rhesus monkey mothers. Asterisk indicates $P<0.05$ and $* *$ indicates $P<0.01$. 

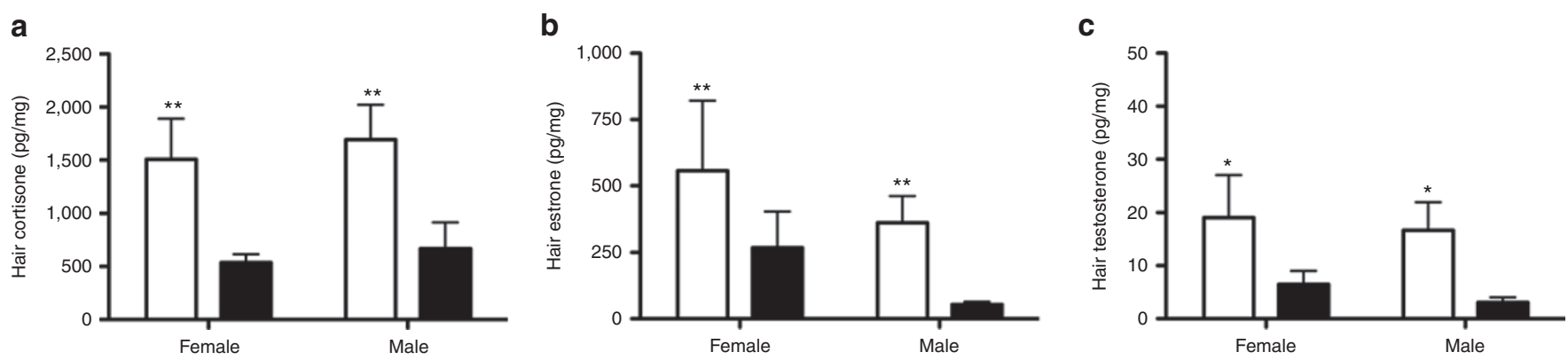

Figure 2. Hair hormone levels in rhesus monkey infants. Hair (mean \pm SEM) cortisone (a), estrone (b), and testosterone (c) in infants born to primiparous ( $n=19$; open bar) and multiparous ( $n=16$; closed bar) rhesus monkey mothers. Asterisk indicates $P<0.05$ and ${ }^{*}$ indicates $P<0.01$ between primiparous and multiparous infants.

Table 1. Steroid hormone levels (mean \pm SD) obtained from the hair of newborn $(n=35)$ and postpartum $(n=35)$ rhesus macaques

\begin{tabular}{lccc}
\hline Hair hormones $(\mathrm{pg} / \mathrm{mg})$ & $\begin{array}{c}\text { Postpartum } \\
\text { mothers }\end{array}$ & $\begin{array}{c}\text { Newborn } \\
\text { infants }\end{array}$ & P value \\
\hline $\begin{array}{l}\text { Glucocorticoids } \\
\quad \text { Cortisol }\end{array}$ & $130.4 \pm 46.53$ & $1,093 \pm 340.0$ & $<0.0001$ \\
$\quad$ Cortisone & $454.5 \pm 240.3$ & $1,360 \pm 1,365$ & $<0.001$ \\
Estrogens & $2.27 \pm 12.5$ & $12.48 \pm 23.3$ & $<0.05$ \\
$\quad \begin{array}{l}\text { Estradiol } \\
\text { Estrone }\end{array}$ & $17.09 \pm 17.85$ & $388.0 \pm 675.0$ & $<0.01$ \\
Androgens & $2.30 \pm 2.5$ & $20.34 \pm 42.6$ & $<0.05$ \\
$\quad \begin{array}{l}\text { Testosterone } \\
\text { Dihydrotestosterone }\end{array}$ & $198.7 \pm 136.4$ & $249 \pm 276.4$ & $0.34, \mathrm{NS}$ \\
$\quad$ Dehydroepiandrosterone & $99.55 \pm 84.5$ & $1,509 \pm 1,236$ & $<0.0001$ \\
Progestin & & & \\
$\quad$ Progesterone & $9.61 \pm 6.8$ & $57.84 \pm 35.8$ & $<0.0001$ \\
\hline $\begin{array}{l}\text { NS, not significant. } \\
P \text { value indicates significance of difference between infants and mothers. }\end{array}$ &
\end{tabular}

progesterone in a single hair specimen. The concentrations of most hormones were dramatically higher, ranging from 5 - to 20 -fold, in the hair of newborn monkeys compared to their mothers (Table 1), indicating that infant hair is reflective of sustained exposure to the high hormone levels present in the fetal compartment during gestation.

\section{Sex Differences in Hair Hormone Levels in Newborn Rhesus Monkeys}

Dehydroepiandrosterone (DHEA) levels were also markedly higher in infant than maternal hair $(P<0.001)$ and significantly higher in the hair of female than male infants $(P<$ 0.05 ; Figure 3 ). DHEA is predominately synthesized by the fetal adrenal gland and serves as a primary substrate for the placental synthesis of estrogens during pregnancy. Although there was a trend for higher DHEA in females born to primiparous dams, variability in values prevented this difference from attaining statistical significance. There were no other significant sex differences in the hair hormone levels for these infants, nor did the hormone profiles of maternal hair reflect whether she was gestating a male or female infant.

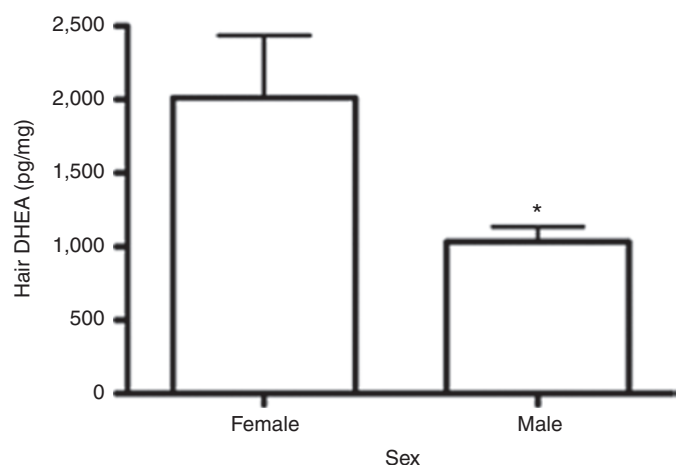

Figure 3. Hair dehydroepiandrosterone (DHEA; mean \pm SEM) in female $(n=15)$ and male $(n=20)$ infants born to primiparous and multiparous rhesus monkey mothers. Asterisk indicates $P<0.05$ between females and males.

\section{DISCUSSION}

This study is the first to demonstrate that a comprehensive panel of steroid hormones can be determined from the hair of a recently born rhesus monkey infant, providing a retrospective reflection of in utero hormone exposure during the final months of gestation. The collection and analytic techniques are noninvasive and could be accomplished with a small lock of head hair in humans. In the fetal monkey, scalp hair begins growing around day 100 of gestation and thus this new hair incorporates hormones in the fetal compartment and amniotic fluid for $\sim 2$ mo until term (14). Hair present on a human infant at birth also begins to appear during the third trimester (20). Assessment of hair hormones in this way also enabled the novel discovery that many steroid hormones were significantly higher in infants born to primigravidae as compared to ones gestated by multiparous females.

Our ability to successfully detect eight hormones in hair from a single injection utilizing high-pressure liquid chromatography-tandem mass spectrometry is another innovative accomplishment, because it offered a more complete view of steroid synthesis and metabolism. Multianalyte techniques have been applied previously to serum (22), urine (23), and feces (24), but this study is the first validation of simultaneous multisteroid measurement from hair. Most research with hair focuses on a single hormone, such as cortisol, and therefore does not take into account that other glucocorticoids, including cortisone, are present at much higher concentrations. During 
pregnancy and fetal development, secondary hormones such as cortisone and estrone are predominant, reflecting placental synthesis and conversion, and are believed to have significant functions. Future studies extending these mass spectrometry techniques are planned to identify and measure other hormones and metabolites in the steroidogenic pathways.

The mechanism by which steroids are incorporated into hair is incompletely understood. During the growing phase of the hair follicle, the free (unbound) fraction of hormone present in blood can diffuse into the growing hair shaft $(6,9,21,25)$. Thus, the analytes in the hair may reflect systemic levels, which can be measured over the length of the hair shaft, or be used to track specific windows of time when the hair shaft is segmented (21). However, interpreting a hair hormone profile is more complicated because of the local steroidogenic potential of cells found at the hair follicle. Sebocytes (sebum-producing epithelial cells) possess key steroidogenic enzymes (26) and the mammalian hair follicle keratinocytes can even synthesize glucocorticoids in situ (27). Sebocytes and keratinocytes are also androgen-sensitive and account for the majority of androgen metabolism in the skin (28). It is likely that some hormones produced by these cells are also incorporated into hair. Therefore, concentrations in hair possibly reflect several sources, not just the levels in systemic circulation.

Nevertheless, prior research on young and adult monkeys has demonstrated that hair cortisol levels are correlated with both blood and saliva levels over a 3-mo period of time (6). Similarly, hair samples collected from gravid women were found to be reflective of rising cortisol levels across the three trimesters of pregnancy (5). Individual differences in hair cortisol levels were also shown to correlate with variation in cortisol excreted during 24-h urine collections in humans (29). Finally, by administering radiolabeled cortisol into guinea pigs, it was possible to demonstrate that $12 \%$ was directly incorporated into hair, although the majority was sequestered as radiolabeled cortisone in the hair (9). The latter result concurs with our data and other papers highlighting the importance of determining cortisone when interested in glucocorticoid activity (30).

In general, the hormone concentrations determined by mass spectrometry were comparable to previously reported values generated with other methods. Hair cortisol levels of adult monkeys was reported to be $50-250 \mathrm{pg} / \mathrm{mg}$ when determined by enzyme-linked immunosorbent assay (6), similar to our mean maternal level of $130 \mathrm{pg} / \mathrm{mg}$. The monkeys' hair testosterone concentrations were also comparable to reported values for humans (31). Hair progesterone levels in women were measured to be $\sim 7.5 \mathrm{pg} / \mathrm{mg}(10)$, which matched the values found in monkey females at delivery. The estrogen levels in the monkeys tended to be lower than reported humans values (32), but may partially reflect the predominant placental synthesis of estrone during pregnancy (33), and local oxidation to estrone because $17 \beta-\mathrm{HSD} 2$ is present in sebaceous glands (34). A marked species difference was evident, however, for the hair concentrations of DHEA, which were much higher in the monkeys than reported for humans $(4.3-5.3 \mathrm{pg} / \mathrm{mg}$, but that too may reflect the high levels of adrenal androgens secreted during pregnancy (33)).
The most dramatic finding was the significantly higher hormone levels present in infant hair as compared to the mothers, and the fact that most steroid hormones were at higher levels in the infants from primiparous pregnancies. Infants from a first-time pregnancy were thus exposed to significantly more estrone and testosterone, two hormones typically associated with sexual differentiation. It was surprising that the levels of estrogens and androgens were not more clearly different between male and infant females, but that may reflect the hormones captured in hair at the end of gestation, whereas sexual differentiation is known to commence between months 1 and 2 after conception in the fetal monkey. In addition, while testosterone and dihydrotestosterone (DHT) have been reported to be higher in the blood of male monkey fetuses, levels of plasma estradiol and estrone were shown not to differ in the fetal circulation of male and female rhesus monkeys, except at day 150 of gestation $(35,36)$. The one hormone in hair that clearly differentiated male and female infants was DHEA, which derives primarily from the fetal adrenal, enabling the placental synthesis of estrogens during pregnancy, especially estrone.

Although further research is needed to verify similar parity effects in humans, there is already some evidence that several placental hormones are higher in the first trimester of primiparous women, and a recent study found higher blood cortisol levels in first-time mothers during the second and third trimester (37). This effect on adrenal activity may reflect the synergistic effect of both the physical demands of pregnancy and perhaps the fact that younger monkeys are more behaviorally reactive during their first pregnancy. It is known that both acute and chronic stressors can affect not only maternal hormone profiles, but also influence hormones associated with sexual differentiation of the fetus.

In conclusion, our research has refined an innovative methodology for investigating hormone synthesis and exposure during late pregnancy, which can be readily utilized in the clinical setting with humans. In addition, the novel discovery is that the young, primiparous female provides a very different endocrine milieu for her infant than does the older, multiparous dam, at least in monkeys. Further evaluations can now be directed toward determining if this exposure to higher levels of steroid hormone during a critical stage of fetal development translates into gender differences in endocrine function and behavior during postnatal life.

\section{METHODS}

\section{Animals}

Mother and infant rhesus monkeys (Macaca mulatta) from a large, long-established breeding colony at the Harlow Primate Laboratory (Madison, WI) were evaluated. The adult females were laboratoryreared primiparous and multiparous adults, 3.9-18.7 y of age. Each was bred with one male for 4-7 d to verify paternity and date of conception. All were descendants of rhesus monkeys originally imported from India, 10-12 generations earlier in the 1950s and 1960s. After pregnancy was confirmed by the cessation of menstruation, the females were housed under standardized conditions until the birth of their infants. The monkeys were continuously in visual and auditory communication with other animals, and an enrichment husbandry program provided each mother with daily stimulation using foraging devices and other manipulanda. However, with the exception of cage 
cleaning, they were not disturbed. All 35 infants, 15 females and 20 males, were born naturally at term. All procedures were approved by the Institutional Animal Care and Use Committee at the University of Wisconsin, Madison.

\section{Parity}

Primiparous $(n=19)$ and multiparous $(n=16)$ adult females and their infants were studied. The mean age of the primiparous females was $4.94 \pm 0.7$ y. Multiparous females were $10.23 \pm 4.5 \mathrm{y}$ of age. The number of prior pregnancies for the multiparous females ranged from 1 to 8 .

\section{Hair Collection}

For hair collection, adult female monkeys and their infants were removed from their cages and infants briefly separated for $\sim 15 \mathrm{~min}$ from their mothers. Hair from the mother was shaved from the upper back region, and hair from infants obtained from the posterior region of the neck and the upper center back. Hair was collected with commercial grooming clippers between 0900 and 1100 (6). Immediately after hair collection, the mother and infant were reunited, and returned to their cage. Hair samples were placed into small aluminum foil pouches for storage at room temperature until ground and assayed.

\section{Chemicals and Reagents}

Steroid reference materials were obtained from Sigma-Aldrich (St Louis, MO): estrone, 17ß0-estradiol, testosterone, DHT, DHEA, progesterone, cortisol, and cortisone. The deuterated internal standards, d5-estradiol, d5-testosterone, d4-DHT, d9-progesterone, and d4-cortisol were obtained from CDN Isotopes (Point-Claire, Quebec, Canada). High-pressure liquid chromatography grade methanol, 2-propanol, water, and formic acid were purchased from Sigma.

\section{Hair Wash Procedure}

Hair washing was conducted as previously described with minor modifications (6). Hair samples of $200 \mathrm{mg}$ were placed in $9 \mathrm{ml}$ polypropylene tubes. Five milliliters of 2-propanol were added to the tube and vortexed for $8 \mathrm{~min}$. After the vortex, $2 \mathrm{ml}$ of the 2-propanol were collected to determine steroid lost during the washing procedure. The wash was repeated and a second $2 \mathrm{ml}$ aliquot of 2-propanol added to the first. The hair was dried in a water bath under a stream of air. Hair was stored in the polypropylene tubes in the dark until processing.

\section{Steroid Extraction}

The steroid extraction method was adapted from previously published studies $(6,30,38)$. Hair was ground to a fine powder using a Retsch ball mill (mixer mill MM 200; $10 \mathrm{ml}$ stainless steel grinding jars; single $12 \mathrm{~mm}$ stainless steel grinding balls) for $5 \mathrm{~min}$ at $30 \mathrm{~Hz}$. Exactly $50 \mathrm{mg}$ of powdered hair was weighed out and carefully placed into a $5 \mathrm{ml}$ glass culture tube. Ground hair was stored in the dark at room temperature until extraction.

For the extraction, methanol $(1 \mathrm{ml})$ and Sorenson buffer $(1 \mathrm{ml})$ were added to each culture tube. The internal standard mixture $(100 \mu \mathrm{l}$ out of $200 \mathrm{pg} / \mu \mathrm{l}$ ) was added to the culture tube. The tube was capped and incubated at $30{ }^{\circ} \mathrm{C}$ for $16 \mathrm{~h}$. After incubation, the tubes were vortexed and centrifuged $(1,500 \mathrm{rpm}, 10 \mathrm{~min})$.The supernatant was removed and run through solid-phase, followed by liquid-phase extraction. For the solid-phase extraction, extraction columns (Waters, Milford, MA) were activated with $1 \mathrm{ml}$ of methanol, followed by $1 \mathrm{ml}$ of water. The sample was deposited and then rinsed with a sequence of $1 \mathrm{ml}$ acetone/deionized water $(2: 8, \mathrm{v} / \mathrm{v}), 1 \mathrm{ml}$ deionized water and $1 \mathrm{ml}$ of hexane. The columns were dried for $30 \mathrm{~min}$ and then the steroids were eluted with three successive aliquots of methanol ( $500 \mu \mathrm{l}$ each). The eluate was evaporated to dryness and then re-suspended with $0.5 \mathrm{ml}$ $\mathrm{NaOH}$. For the liquid-liquid extraction, $3 \mathrm{ml}$ ethyl acetate were added to the reconstituted sample, the tube was vortexed for $8 \mathrm{~min}$, and then centrifuged ( $3 \mathrm{~min}, 1,000 \mathrm{rpm}$ ). The organic phase was removed and stored at $4{ }^{\circ} \mathrm{C}$ until analysis. Immediately prior to analysis, samples were evaporated to dryness in a water bath and then resuspended in $40 \mu \mathrm{l}$ of 20:80 methanol/water.

\section{Analytical Procedure}

All samples were analyzed on a QTRAP 5500 quadrupole linear ion trap mass spectrometer (AB Sciex, Concord, ON, Canada) equipped with an atmospheric pressure chemical ionization source. The system included two Shimadzu LC20ADXR pumps and a Shimadzu SIL20ACXR autosampler.

The chromatographic separation was performed on Phenomenex Kinetex 2.6u C18 100A, $150 \times 4.6 \mathrm{~mm}$ column (Phenomenex, Torrance, CA). The mobile phase consisted of two solvents: water (A) and methanol (B) delivered at a flow rate of $0.25 \mathrm{ml} / \mathrm{min}$. The sample $(10 \mu \mathrm{l})$ was injected into the LC/MS/MS after which the injector was washed with three postinjection washes with 20:80 methanol:water. The total run time was $40 \mathrm{~min}$. During the first $2 \mathrm{~min}$, the initial condition of $3 \% \mathrm{~B}$ were held. This was followed by fast linear gradient to $50 \% \mathrm{~B}$ over the next $0.10 \mathrm{~min}$. These system conditions were maintained for the next $2.9 \mathrm{~min}$. The gradient was increased to $67 \% \mathrm{~B}$ over the next $15 \mathrm{~min}$, then $100 \% \mathrm{~B}$ over the next $3 \mathrm{~min}$. This was held for $7 \mathrm{~min}$ before the system was returned to initial conditions of $3 \% \mathrm{~B}$ over $0.1 \mathrm{~min}$ and held for the final $9.9 \mathrm{~min}$ of each run.

Once ion pairs were selected for each of the compounds and the internal standards, the instrument setting were optimized for maximal signal using the autotune feature of the instrument software. Results were generated in positive-ion mode with the following optimized voltages: corona discharge current: $3 \mathrm{~V}$, entrance potential: $10 \mathrm{~V}$. The source temperature was $500{ }^{\circ} \mathrm{C}$. The gas settings were: curtain gas: 20 psi, nebulizing gas: $40 \mathrm{psi}$, collisionally activated dissociation gas: medium.

Quantitative results were recorded as multiple reaction monitoring area counts after determination for the response factor for each compound and internal standard. Internal standard pairs were as follows: d4 estrone with estrone, d5 estradiol with estradiol, d5 testosterone with testosterone, DHT and DHEA, d9 progesterone with progesterone, $\mathrm{d} 4$ cortisol with cortisol and cortisone.

All data were acquired and processed with Analyst software, Ver 1.5.1 (AB-Sciex Concord, ON). The detection limit were established as the lowest analyte concentrations that could be distinguished from the absence of analyte with $>95 \%$ certainty based on 10 replicate experiments each on low amounts of added compounds. The limit of quantification were arbitrarily set at the lowest analyte concentration that could be measured in actual hair samples with an interassay coefficient of variance $<20 \%$.

Eight-point standard calibration curves were obtained by using $1 \mathrm{mg}$ of melanin, which is the main constitutive part of hair influencing the incorporation of xenobiotics $(30,39,40)$. The mean concentration of melanin in $100 \mathrm{mg}$ of human hair is $1 \mathrm{mg}$. Because $50 \mathrm{mg}$ of hair were analyzed in the present study, $0.5 \mathrm{mg}$ of melanin was added to each concentration level. The final concentrations of the calibration curves were: $15.6,31.3,62.5,125,250,500,1,000$, and 2,000 pg/tube. The linearity for all was $r>0.9990$ and the curve fit was linear through zero with $1 / \times$ weighting. For DHT only, the curve fit was linear, with $1 / \times$ weighting. None of the compounds of interest were detected in zero or blank samples.

To assess recovery, the peak areas of individual analytes in extracted samples were assessed with the analyte of the same concentration that was not extracted, but simply dried down and reconstituted in the injection solvent. The recovery ranged from 77 to $103 \%$, with the glucocorticoids displaying the highest recovery and progesterone the lowest.

Intraassay precision was determined by extracting and assaying nine separate aliquots of a ground hair pool from many monkeys in a single batch. The intraassay coefficient of variance ranged from 2 to $9 \%$. Interassay precision was determined by testing the pool in five assays. The interassay coefficient of variance ranged from 8 to $17 \%$.

\section{Statistics}

Unpaired $t$-tests were used to verify the significance of differences in hair hormone levels between infants from primiparous and multiparous mothers, and between male and female infants. Two-way ANOVAs (pregnancy condition $\times$ infant sex) were used to test the differential influence of parity on male and female infants. The Pearson test was employed to examine the association between maternal and infant values. When variances were found to differ significantly, data were log-transformed and reanalyzed. Outliers were detected using the extreme studentized deviate. All statistical analyses were completed using GraphPad Prism (San Diego, CA). Statistical significance was defined as $P<0.05$ for all tests. 


\section{ACKNOWLEDGMENTS}

This study was supported by National Institutes of Health grants (R01 HD057064 and P01 HD39386); a Grand Challenges Exploration award from the Bill and Melinda Gates Foundation to CLC; as well as resources from the Wisconsin National Primate Research Center (P51OD11106-52), the University of Wisconsin Institute for Clinical and Translational Research (UL1TR000427), and the Wisconsin State Laboratory of Hygiene.

\section{REFERENCES}

1. Bai J, Wong FW, Bauman A, Mohsin M. Parity and pregnancy outcomes. Am J Obstet Gynecol 2002;186:274-8.

2. Beard JL. Iron deficiency: assessment during pregnancy and its importance in pregnant adolescents. Am J Clin Nutr 1994;59:Suppl 2:502S-508S discussion 508S-510S.

3. Järvelä IY, Zácková T, Laitinen P, Ryynänen $M$, Tekay A. Effect of parity and fetal sex on placental and luteal hormones during early first trimester. Prenat Diagn 2012;32:160-7.

4. Zuppa AA, Tornesello A, Papacci P, et al. Relationship between maternal parity, basal prolactin levels and neonatal breast milk intake. Biol Neonate 1988;53:144-7.

5. D’Anna-Hernandez KL, Ross RG, Natvig CL, Laudenslager ML. Hair cortisol levels as a retrospective marker of hypothalamic-pituitary axis activity throughout pregnancy: comparison to salivary cortisol. Physiol Behav 2011;104:348-53

6. Davenport MD, Tiefenbacher S, Lutz CK, Novak MA, Meyer JS. Analysis of endogenous cortisol concentrations in the hair of rhesus macaques. Gen Comp Endocrinol 2006;147:255-61.

7. Phillips DI, Barker DJ, Fall CH, et al. Elevated plasma cortisol concentrations: a link between low birth weight and the insulin resistance syndrome? J Clin Endocrinol Metab 1998;83:757-60.

8. O'Connor TG, Heron J, Golding J, Beveridge M, Glover V. Maternal antenatal anxiety and children's behavioural/emotional problems at 4 years. Report from the Avon Longitudinal Study of Parents and Children. Br J Psychiatry 2002;180:502-8.

9. Keckeis K, Lepschy M, Schöpper H, Moser L, Troxler J, Palme R. Hair cortisol: a parameter of chronic stress? Insights from a radiometabolism study in guinea pigs. J Comp Physiol B, Biochem Syst Environ Physiol 2012;182:985-96.

10. Yang HZ, Lan J, Meng YJ, Wan XJ, Han DW. A preliminary study of steroid reproductive hormones in human hair. J Steroid Biochem Mol Biol 1998;67:447-50.

11. Wallen K. The Organizational Hypothesis: Reflections on the $50^{\text {th }}$ anniversary of the publication of Phoenix, Goy, Gerall, and Young (1959). Horm Behav 2009;55:561-5.

12. Crews D, Bull JJ. Sex determination: some like it hot (and some don't). Nature 2008;451:527-8.

13. Cariello MO, Macedo RH, Schwabl HG. Maternal androgens in eggs of communally breeding guira cuckoos (Guira guira). Horm Behav 2006;49:654-62.

14. Schultz A. Fetal growth and development of the rhesus monkey. Embryology 1937;155:86-7.

15. Daniel CR 3rd, Piraccini BM, Tosti A. The nail and hair in forensic science. J Am Acad Dermatol 2004;50:258-61.

16. You Y, Uboh CE, Soma LR, et al. Simultaneous separation and determination of 16 testosterone and nandrolone esters in equine plasma using ultra high performance liquid chromatography-tandem mass spectrometry for doping control. J Chromatogr A 2011;1218:3982-93.

17. Bencko V. Use of human hair as a biomarker in the assessment of exposure to pollutants in occupational and environmental settings. Toxicology 1995;101:29-39.

18. Sachs H. Quality control by the Society of Hair Testing. Forensic Sci Int 1997;84:145-50.
19. Aleksa K, Liesivuori J, Koren G. Hair as a biomarker of polybrominated diethyl ethers' exposure in infants, children and adults. Toxicol Lett 2012;210:198-202.

20. Forman R, Schneiderman J, Klein J, Graham K, Greenwald M, Koren G. Accumulation of cocaine in maternal and fetal hair; the dose response curve. Life Sci 1992;50:1333-41.

21. Stalder T, Kirschbaum C. Analysis of cortisol in hair-state of the art and future directions. Brain Behav Immun 2012;26:1019-29.

22. Rothman MS, Carlson NE, Xu M, et al. Reexamination of testosterone, dihydrotestosterone, estradiol and estrone levels across the menstrual cycle and in postmenopausal women measured by liquid chromatography-tandem mass spectrometry. Steroids 2011;76:177-82.

23. Hauser B, Deschner T, Boesch C. Development of a liquid chromatography-tandem mass spectrometry method for the determination of 23 endogenous steroids in small quantities of primate urine. J Chromatogr B Analyt Technol Biomed Life Sci 2008;862:100-12.

24. Weltring A, Schaebs FS, Perry SE, Deschner T. Simultaneous measurement of endogenous steroid hormones and their metabolites with LC-MS/MS in faeces of a New World primate species, Cebus capucinus. Physiol Behav 2012;105:510-21.

25. Cone EJ. Mechanisms of drug incorporation into hair. Ther Drug Monit 1996;18:438-43.

26. Thiboutot D, Jabara S, McAllister JM, et al. Human skin is a steroidogenic tissue: steroidogenic enzymes and cofactors are expressed in epidermis, normal sebocytes, and an immortalized sebocyte cell line (SEB-1). J Invest Dermatol 2003;120:905-14.

27. Slominski A. A nervous breakdown in the skin: stress and the epidermal barrier. J Clin Invest 2007;117:3166-9.

28. Fritsch M, Orfanos CE, Zouboulis CC. Sebocytes are the key regulators of androgen homeostasis in human skin. J Invest Dermatol 2001;116:793-800.

29. Sauvé B, Koren G, Walsh G, Tokmakejian S, Van Uum SH. Measurement of cortisol in human hair as a biomarker of systemic exposure. Clin Invest Med 2007;30:E183-91.

30. Raul JS, Cirimele V, Ludes B, Kintz P. Detection of physiological concentrations of cortisol and cortisone in human hair. Clin Biochem 2004;37:1105-11.

31. Scherer C, Wachter U, Wudy SA. Determination of testosterone in human hair by gas chromatography-selected ion monitoring mass spectrometry. Analyst 1998;123:2661-3.

32. Choi MH, Kim KR, Chung BC. Determination of estrone and 17 betaestradiol in human hair by gas chromatography-mass spectrometry. Analyst 2000;125:711-4.

33. Pepe GJ, Albrecht ED. Actions of placental and fetal adrenal steroid hormones in primate pregnancy. Endocr Rev 1995;16:608-48.

34. Thiboutot D, Martin P, Volikos L, Gilliland K. Oxidative activity of the type 2 isozyme of 17beta-hydroxysteroid dehydrogenase (17betaHSD) predominates in human sebaceous glands. J Invest Dermatol 1998;111:390-5.

35. Resko JA, Pleom JG, Stadelman HL. Estrogens in fetal and maternal plasma of the rhesus monkey,. Endocrinology 1975;97:425-30.

36. Resko JA, Ellinwood WE, Pasztor LM, Huhl AE. Sex steroids in the umbilical circulation of fetal rhesus monkeys from the time of gonadal differentiation. J Clin Endocrinol Metab 1980;50:900-5.

37. Bouyou-Akotet MK, Adegnika AA, Agnandji ST, et al. Cortisol and susceptibility to malaria during pregnancy. Microbes Infect 2005;7:1217-23.

38. Cirimele V, Kintz P, Dumestre V, Goullé JP, Ludes B. Identification of ten corticosteroids in human hair by liquid chromatography-ionspray mass spectrometry. Forensic Sci Int 2000;107:381-8.

39. Wennig R. Potential problems with the interpretation of hair analysis results. Forensic Sci Int 2000;107:5-12.

40. Kronstrand R, Förstberg-Peterson S, Kågedal B, Ahlner J, Larson G. Codeine concentration in hair after oral administration is dependent on melanin content. Clin Chem 1999;45:1485-94. 